\title{
RESÍDUOS DE SERRARIAS NA PRODUÇÃO DE PAINÉIS DE MADEIRA AGLOMERADA DE EUCALIPTO
}

\section{EUCALYPTU'S WOOD RESIDUES DERIVING FROM SAWMILLS FOR PARTICLEBOARD MANUFACTURING}

\author{
Setsuo IWAKIRI $\varliminf^{\circ}$ \\ Alexandro Bayestorf da CUNHA ${ }^{2}$ \\ Carlos Eduardo Camargo ALBUQUERQUE ${ }^{3}$ \\ Elianice GORNIAK² \\ Lourival Marin MENDES ${ }^{3}$
}

\begin{abstract}
RESUMO
Esta pesquisa foi desenvolvida com o objetivo de avaliar a viabilidade de utilização da madeira de Eucalyptus maculata, E. grandis e E. tereticornis, na forma de resíduos de processamento em serrarias, para produção de painéis de madeira aglomerada. Foram produzidos 24 painéis, para um total de oito tratamentos definidos em função de três espécies e mistura proporcional destas e dois níveis de resina ( 8 e 12\%). Os painéis foram produzidos com densidade nominal de $0,75 \mathrm{~g}_{\mathrm{cm}} \mathrm{cm}^{-3}$ e de acordo com o seguinte ciclo de prensagem: temperatura de prensagem $=140^{\circ} \mathrm{C}$, tempo de prensagem $=8 \mathrm{~min}$ e pressão específica $=40 \mathrm{~kg} \cdot \mathrm{cm}^{-2}$. As seguintes propriedades físico-mecânicas dos painéis foram analisadas: absorção de água (AA-2 e 24 h), inchamento em espessura (IE-2 e 24 h), ligação interna (LI), módulo de elasticidade (MOE) e módulo de ruptura (MOR) em flexão estática. Os resultados dos ensaios indicaram que os painéis produzidos com a madeira destas espécies de eucalipto apresentam propriedades físico-mecânicas satisfatórias, em comparação com algumas espécies referenciais como Pinus elliottii, Mimosa scabrella e Eucalyptus dunnii, mencionadas na literatura. Os painéis produzidos com $12 \%$ de resina apresentaram melhores resultados que os painéis com $8 \%$. Com base nas propriedades mecânicas, o Eucalyptus maculata e o Eucalyptus grandis foram as espécies que apresentaram melhor comportamento para produção de painéis de madeira aglomerada.
\end{abstract}

Palavras-chave: painéis de madeira aglomerada, eucalipto, resíduos de serraria.

\begin{abstract}
This research paper was developed aiming at evaluating the feasibility of using Eucalyptus maculata, E. grandis and E. tereticornis woods, derived from saw mill processing residues to particleboard manufacture. Was manufactured 24 boards, being eight treatments, defined in function of three species and its mixture, and two resin levels ( 8 and $12 \%)$. The processing variables were: board density $=0,75 \mathrm{~g} \cdot \mathrm{cm}^{-3}$, press temperature $=140^{\circ} \mathrm{C}$, pressing time $=8 \mathrm{~min}$ and pressure $=40$ $\mathrm{kg} . \mathrm{cm}^{-2}$. The following physical and mechanical properties were evaluated: water absorption (AA-2 and $24 \mathrm{~h}$ ), thickness swelling (IE-2 and IE24 h), internal bond (LI), modulus of elasticity (MOE) and modulus of rupture (MOR) in bending strenght. Boards manufactured with these eucaliptu's species showed satisfactory physical and mechanical properties in comparison to some referential species such as Pinus elliottii, Mimosa scabrella and Eucalyptus dunnii presented in the literature. The boards manufactured with $12 \%$ of resin level showed better results than boards with $8 \%$. Based in the mechanical properties, the Eucalyptus maculata showed the best behavior for particleboard manufacture.
\end{abstract}

Key-words: particleboard, eucaliptus, wood waste.

\footnotetext{
${ }^{1}$ Engenheiro Florestal, Doutor, Professor Titular, Universidade Federal do Paraná. E-mail: setsuo@cwb.matrix.com.br $\Xi^{\circ}$ Autor para correspondência.

2 Engenheiro Florestal, Mestrando em Engenharia Florestal - Universidade Federal do Paraná.

${ }^{3}$ Engenheiro Florestal, Doutorando em Engenharia Florestal - Universidade Federal do Paraná.
} 


\section{INTRODUÇÃO}

As indústrias de base florestal no Brasil geram um volume significativo de resíduos durante as fases operacionais, desde a exploração florestal até o produto final. De acordo com Brito (1996), as indústrias de madeira serrada, lâminas e compensados geraram resíduos da ordem de $19.255 .000 \mathrm{~m}^{3}$.ano-1 , na forma de costaneiras, refilos, aparas, cascas, serragem, cepilhos ou maravalhas etc., representando $50,7 \%$ do volume original de toras.

Uma parte substancial de resíduos provenientes de processamento mecânico da madeira é utilizada para geração de energia para fins industriais e domésticos. No entanto, apesar dos benefícios sociais, esta forma de utilização agrega muito pouco valor ao produto final, sendo necessárias outras formas alternativas de utilização.

O aproveitamento de resíduos na forma de costaneiras, refilos e aparas, provenientes de serrarias, para produção de painéis de madeira aglomerada, seria uma das alternativas, no sentido de se obter um produto de maior valor agregado. $O$ posterior revestimento superficial dos painéis, por colagem de lâminas naturais ou sintéticas, para utilização na indústria moveleira, elevaria mais ainda este valor agregado.

A primeira indústria de aglomerado no Brasil foi instalada em Curitiba, no ano de 1966, e conta atualmente com mais de dez unidades fabris, distribuídas nas regiões Sul e Sudeste do País. A quase totalidade da madeira utilizada na produção de painéis de madeira aglomerada é proveniente de reflorestamentos de pinus, espécie que é também utilizada para produção de polpa e papel, madeira serrada e beneficiada, lâminas para painéis compensados e, mais recentemente, para produção de painéis de fibras de média densidade (MDF).

Diante da demanda crescente pela madeira de pinus surge, também, a necessidade de utilização de espécies alternativas de reflorestamento, tais como as inúmeras espécies de eucalipto disponíveis em grandes áreas de plantios comerciais e experimentais.

Uma das principais variáveis de processo na produção de painéis de madeira aglomerada é a espécie da madeira e a conseqüente relação entre a densidade do painel e a densidade da madeira utilizada, denominada de razão de compactação (Kelly, 1977; Moslemi, 1974; Maloney, 1993; Tsoumis, 1991). Os autores recomendam que a razão de compactação deve estar na faixa de 1,3 a 1,6 para que ocorra adequada densificação e consolidação do painel à espessura final desejada. Segundo Kawai e Sassaki (1993), as propriedades mecânicas dos painéis de madeira aglomerada apresentam uma relação direta e linear com a razão de compactação. Para o caso especial de colagem de partículas de maior área superficial com resina isocianato, o limite mínimo pode chegar a 0,7. Em termos de estabilidade dimensional, os autores afirmam que o inchamento em espessura do painel de média densidade é maior com o aumento na razão de compactação e menor para painéis de baixa densidade, independente da espécie utilizada e da razão de compactação. Por outro lado, a baixa densidade do painel pode favorecer 0 ataque de fungos, causando redução principalmente na resistência à flexão estática (Imamura, 1993).

As espécies de maior densidade podem ser utilizadas em mistura com as de menor densidade, em proporções variáveis, conforme a magnitude da diferença em densidade entre as espécies (Moslemi, 1974; Maloney, 1993). El-Osta (1994) avaliou a influência de diferentes proporções de mistura de madeira das espécies Casuarina glauca e Salix safsaf e constatou que, com a proporção de 1:1, as propriedades de flexão estática, ligação interna, inchamento em espessura e absorção de água melhoraram em comparação aos painéis produzidos com espécies individualmente.

Pesquisas realizadas por Iwakiri et al. (1996), para painéis produzidos com mistura de madeiras de Pinus elliottii e Eucalyptus dunnii, demonstraram que com o aumento na razão de compactação, em função da maior proporção de pinus no painel, resulta em aumento nos valores médios de absorção de água e inchamento em espessura. Por outro lado, a resistência à flexão estática não foi influenciada significativamente com a mistura de duas espécies no painel. Na pesquisa desenvolvida com a mistura de madeiras de Pinus elliottii e Mimosa scabrella, Iwakiri et al. (1995) concluíram que a mistura destas espécies, em proporção de $50 \%$ cada, resulta em painéis com melhores propriedades físicomecânicas. Os autores ressaltam a importância destes resultados, tendo em vista a viabilidade de melhor aproveitamento da madeira de Mimosa scabrella, utilizada principalmente como lenha e carvão, em produto de maior valor agregado.

Perante o exposto, o presente trabalho foi desenvolvido com o objetivo de avaliar a viabilidade de utilização da madeira de Eucalyptus maculata, Eucalyptus grandis e Eucalyptus tereticornis, na forma de resíduos de processamento em serrarias, para produção de painéis de madeira aglomerada.

\section{METODOLOGIA}

Os materiais utilizados para a produção de painéis de madeira aglomerada foram costaneiras obtidas do desdobro primário de toras em serraria, localizada na Estação Experimental do Cangüiri, da Universidade Federal do Paraná, no Município de Quatro Barras, Estado do Paraná.

As espécies estudadas foram: Eucalyptus maculata, Eucalyptus grandis e Eucalyptus tereticornis, com 16 anos de idade, provenientes de plantios localizados na fazenda experimental do 
Departamento de Ciências Florestais da Universidade de São Paulo, em Piracicaba, SP.

A resina utilizada para colagem de partículas foi a uréia-formaldeído, com pH 7,2 e teor de sólidos de $55 \%$, com catalisador à base de sulfato de amônia. A partir das costaneiras, as partículas foram geradas com as dimensões nominais de $25 \mathrm{~mm}$ de comprimento, 0,7 $\mathrm{mm}$ de espessura e largura variável, sendo posteriormente reduzidas em partículas menores, por um moinho de martelo. As partículas foram secas para aproximadamente $5 \%$ de teor de umidade, em estufa com circulação de ar forçado à temperatura de $80^{\circ} \mathrm{C} \mathrm{e}$, posteriormente, peneiradas para remoção de "finos".

Os painéis foram produzidos à densidade nominal de $0,75 \mathrm{~g} . \mathrm{cm}^{-3}$, com as dimensões de $45 \mathrm{~cm} \times 55 \mathrm{~cm} \times 1,5 \mathrm{~cm}$. O ciclo de prensagem utilizado foi: temperatura $=140^{\circ} \mathrm{C}$, pressão específica $=40 \mathrm{~kg} \cdot \mathrm{cm}^{-2}$ e tempo de prensagem $=$ $8 \mathrm{~min}$. Foram produzidos 24 painéis com três repetições (chapas) por tratamento. As variáveis de estudo foram: três espécies e mistura proporcional destas, com dois níveis de resina, como demonstrado no delineamento apresentado na Tabela 1.

TABELA 1 - Delineamento experimental.

\begin{tabular}{clc} 
Tratamento & \multicolumn{1}{c}{ Espécie } & $\begin{array}{c}\text { Quantidade } \\
\text { de resina (\%) }\end{array}$ \\
& & Mistura das três espécies \\
1 & Mistura das três espécies & 12 \\
2 & Eucalyptus maculata & 8 \\
3 & Eucalyptus maculata & 12 \\
4 & Eucalyptus grandis & 8 \\
5 & Eucalyptus grandis & 12 \\
6 & Eucalyptus tereticornis & 8 \\
7 & Eucalyptus tereticornis & 12 \\
8 & Eucaly
\end{tabular}

Após a prensagem os painéis foram acondicionados em câmara climática à temperatura de $20 \pm 2^{\circ} \mathrm{C}$ e umidade relativa de $65 \pm 3 \%$, até atingir peso constante, no teor de umidade de equilíbrio em torno de $12 \%$.

Após o acondicionamento foram retirados os corpos de prova para a realização de ensaios físicomecânicos, de acordo com os procedimentos prescritos na norma ASTM D-1037 (1992). Os ensaios realizados foram:

- absorção de água após 2 e 24 horas de imersão;

- inchamento em espessura após 2 e 24 horas de imersão em água;

- ligação interna; e

- flexão estática (módulo de elasticidade e módulo de ruptura).

O delineamento experimental utilizado foi o inteiramente casualizado. Os resultados obtidos foram submetidos à análise de variância. As médias das variáveis identificadas com $\mathrm{F}$ significativo a $5 \%$ de probabilidade foram submetidas à comparação pelo teste de Tukey, também a 5\% de probabilidade.

\section{RESULTADOS E DISCUSSÃO}

\section{Propriedades físicas}

Os valores médios para absorção de água (2 e $24 \mathrm{~h}$ ) e inchamento em espessura (2 e $24 \mathrm{~h}$ ) são apresentados na Tabela 2.

TABELA 2 - Propriedades físicas dos painéis ${ }^{1}$

\begin{tabular}{|c|c|c|c|c|c|c|}
\hline Tratamento $^{2}$ & AA - & $2 \mathrm{~h}(\%)^{3}$ & $A A-24 h(\%)^{3}$ & $\mathrm{IE}-2$ & $h(\%)^{4}$ & $\mathrm{IE}-24 \mathrm{~h}(\%)^{4}$ \\
\hline Mistura - 8 & 34,11 & $b$ & 58,33 a & 25,72 & $a b$ & $35,11 \mathrm{ab}$ \\
\hline Mistura - 12 & 18,29 & $d e$ & 45,59 & 15,55 & $c d$ & 28,37 \\
\hline E. maculata - 8 & 41,74 & & $56,54 a b$ & 30,60 & a & 38,81 a \\
\hline E. maculata - 12 & 27,95 & b c & 50,06 & 21,25 & b c & $32,22 a b$ \\
\hline E. grandis - 8 & 24,75 & $c d$ & 58,80 a & 21,18 & b c & $34,95 \mathrm{ab}$ \\
\hline E. grandis-12 & 13,94 & e & 37,37 & 12,38 & d & 24,23 \\
\hline E. tereticornis - 8 & 21,56 & $c d e$ & 48,05 & 16,76 & $c d$ & 30,10 \\
\hline E. tereticornis - 12 & 15,67 & $\mathrm{de}$ & 37,82 & 12,48 & d & 23,51 \\
\hline
\end{tabular}

1 Médias seguidas da mesma letra são estatisticamente iguais pelo teste de Tukey a $5 \%$ de probabilidade.

28 e 12 são as percentagens da resina.

${ }^{3} \mathrm{AA}=$ Absorção de água (2 e $24 \mathrm{~h}$ ).

${ }^{4} \mathrm{IE}=$ Inchamento em espessura $(2 \mathrm{e} 24 \mathrm{~h})$.

\section{Absorção de água}

Os valores médios de absorção de água, para 2 e 24 h de imersão em água, variaram respectivamente de 13,94 a $41,74 \%$ e de 37,37 a $58,80 \%$. Na pesquisa realizada por Iwakiri et al.
(1995), para painéis de madeira aglomerada de pinus e bracatinga e mistura destas espécies, os valores médios obtidos foram na faixa de 41,31 a $98,96 \%$ para AA-2 h e de 60,98 a $106,65 \%$ para AA 24 h. Os resultados obtidos para painéis produzidos com espécies de Pinus elliottii e 
Eucalyptus dunnii mostraram valores médios de absorção de água que variaram na faixa de 42,09 a $71,75 \%$ para $2 \mathrm{~h}$ de imersão e de 73,12 a $80,05 \%$ para $24 \mathrm{~h}$ (Iwakiri et al., 1996). Portanto, o comportamento das espécies de eucalipto utilizadas nesta pesquisa foi satisfatório em comparação aos painéis de Pinus elliottii, Mimosa scabrella e Eucalyptus dunnii.

Os painéis produzidos com $12 \%$ de resina apresentaram tendências para menor absorção de água em relação aos painéis com $8 \%$ de resina, tanto para $2 \mathrm{~h}$, quanto para $24 \mathrm{~h}$ de imersão. A aplicação de maior quantidade de resina às partículas de madeira pode ter contribuído para melhor impermeabilização das superfícies das partículas e, conseqüentemente, para redução na absorção de água.

Com relação à variável espécie, os painéis produzidos com a madeira de $E$. grandis e $E$. tereticornis foram os que apresentaram menores valores médios de absorção de água, tanto para 2 $\mathrm{h}$ quanto para $24 \mathrm{~h}$ de imersão em água. Os painéis de E. maculata apresentaram comportamento inferior em absorção de água.

\section{Inchamento em espessura}

Os valores médios de inchamento em espessura, para 2 e $24 \mathrm{~h}$ de imersão em água, variaram respectivamente de 12,38 a $30,60 \%$ e de 23,51 a $38,81 \%$. Nas pesquisas anteriores realizadas por Iwakiri et al. (1995) e (1996) para painéis de madeira aglomerada produzidos com as espécies de Pinus elliottii, Mimosa scabrella e Eucalyptus dunnii, os valores médios de inchamento em espessura variaram na faixa de 20,43 a $50,97 \%$ para $2 \mathrm{~h}$ de imersão em água e de 23,87 a 53,41\% para $24 \mathrm{~h}$.

A aplicação de maior quantidade de resina reduziu o inchamento em espessura dos painéis. No entanto, para IE $24 \mathrm{~h}$, apenas os valores para
E.grandis apresentaram diferença estatisticamente significativa. Com relação às espécies, os painéis produzidos com E. grandis e E. tereticornis apresentaram melhor comportamento em inchamento em espessura, tanto para 2 quanto para 24 h de imersão em água.

\section{Propriedades mecânicas}

Os valores médios para ligação interna, módulo de elasticidade e módulo de ruptura em flexão estática são apresentados na Tabela 3.

\section{Ligação interna}

Os valores médios de ligação interna variaram na faixa 7,63 a 13,63 kg. $\mathrm{cm}^{-2}$. Estes valores podem ser considerados satisfatórios em comparação aos resultados obtidos por Iwakiri et al. (1995) e (1996), para painéis produzidos com Pinus elliottiii (11,0 kg.cm-2), Mimosa scabrella (4,48 $\left.\mathrm{kg} \cdot \mathrm{cm}^{-2}\right)$ e Eucalyptus dunnii $\left(8,24 \mathrm{~kg} \cdot \mathrm{cm}^{-2}\right)$.

Os painéis produzidos com $12 \%$ de resina apresentaram maiores valores de ligação interna em relação aos painéis com $8 \%$, principalmente para as espécies $E$. grandis e E. tereticornis, cujas diferenças foram estatisticamente significativas. Este incremento em resistência pode ser atribuído à maior disponibilidade de resina por unidade de área das partículas. Todos os valores médios de ligação interna foram superiores ao valor mínimo de referência exigido pela norma americana CS 23666 , de $4,2 \mathrm{~kg} \cdot \mathrm{cm}^{-2}$.

Para painéis produzidos com $8 \%$ de resina, a espécie que melhor comportou-se, para esta propriedade foi o E. maculata, com $11,09 \mathrm{~kg} . \mathrm{cm}^{-2}$, sendo estatisticamente superior em relação às demais espécies e mistura destas. Por outro lado, os painéis de E. tereticornis produzidos com $12 \%$ de resina apresentaram maior valor médio de ligação interna $\left(13,63 \mathrm{~kg} \cdot \mathrm{cm}^{-2}\right)$.

TABELA 3 - Propriedades mecânicas dos painéis ${ }^{1}$

\begin{tabular}{|c|c|c|c|c|}
\hline Tratamento $^{2}$ & $\mathrm{LI}\left(\mathrm{kg} \cdot \mathrm{cm}^{-2}\right)^{3}$ & $\operatorname{MOE}\left(\mathrm{kg} \cdot \mathrm{cm}^{-2}\right)^{4}$ & MOR & $\left.\mathrm{cm}^{-2}\right)^{5}$ \\
\hline Mistura - 8 & $7,63 \quad d$ & $15.296,08 \quad b c$ & 126,88 & C \\
\hline Mistura - 12 & $12.41 \mathrm{a} \mathrm{b}$ & $18.716,00$ a b c & 158,54 & a \\
\hline E. maculata - 8 & $11,09 a b$ & $18.394,94$ a b c & 134,16 & b c \\
\hline E. maculata -12 & $12,77 \mathrm{ab}$ & $19.960,12 a b$ & 163,36 & a \\
\hline E. grandis - 8 & 7,74 & $22.068,95$ a & 130,79 & c \\
\hline E. grandis-12 & 10,80 & $23.055,65 \mathrm{a}$ & 152,95 & $a b$ \\
\hline E. tereticornis - 8 & 8,49 & $17.737,63$ & 74,13 & e \\
\hline E. tereticornis - 12 & $13,63 \mathrm{a}$ & $12.178,95$ & 105,57 & \\
\hline
\end{tabular}

1 Médias seguidas da mesma letra são estatisticamente iguais pelo teste de Tukey a $5 \%$ de probabilidade.

28 e 12 são as percentagens da resina.

${ }^{3} \mathrm{LI}=$ ligação interna.

${ }^{4} \mathrm{MOE}=$ módulo de elasticidade

${ }^{5} \mathrm{MOR}=$ módulo de ruptura . 


\section{Módulo de elasticidade}

Os valores médios de módulo de elasticidade em flexão estática variaram na faixa de 12.178,95 a $23.055,65 \mathrm{~kg} \mathrm{~cm}^{-2}$. Em pesquisa anterior conduzida por Iwakiri et al. (1995), foi obtido valor médio de $22.691,34 \mathrm{~kg} . \mathrm{cm}^{-2}$ para painéis produzidos com Eucalyptus dunnii.

Com relação aos dois níveis de resina, não foi detectada diferença estatisticamente significativa entre os painéis produzidos com 8 e 12\% de resina. Os painéis de $E$. grandis produzidos com $8 \%$ de resina apresentaram valores médios de $\mathrm{MOE}$ estatisticamente superiores aos painéis de $E$. tereticornis e mistura de espécies. Para $12 \%$ de resina, os painéis de E. tereticornis foram os que apresentaram piores resultados.

\section{Módulo de ruptura}

Os valores médios de módulo de ruptura em flexão estática variaram na faixa de 74,13 a 163,36 $\mathrm{kg} . \mathrm{cm}^{-2}$. O valor médio de MOR obtido para painéis de E. dunnii foi de $169,41 \mathrm{~kg} \cdot \mathrm{cm}^{-2}$ (Iwakiri et al. 1995).

Para todas as espécies e mistura destas, os painéis produzidos com $12 \%$ de resina apresentaram valores de MOR estatisticamente superiores aos painéis produzidos com $8 \%$ de resina.

Tanto para 8 quanto para $12 \%$ de resina, os painéis de E. maculata, E. grandis e mistura de três espécies apresentaram valores médios de MOR estatisticamente superiores em relação aos painéis de E. tereticornis. Entretanto, este resultado não inviabiliza a utilização de E. tereticornis, pois, poderia, em mistura com as duas demais espécies, produzir painéis com maior MOR.

\section{CONCLUSÕES}

1) As espécies de eucalipto estudadas apresentaram menor absorção de água e inchamento em espessura dos painéis, em comparação com as principais espécies referenciais como Pinus elliottii, Mimosa scabrella e Eucalyptus dunnii;

2) Os painéis de Eucalyptus grandis e Eucalyptus tereticornis apresentaram melhor estabilidade dimensional em relação aos painéis de Eucalyptus maculata. A utilização de maior quantidade de resina contribuiu para melhorar a estabilidade dimensional dos painéis;

3) Para todas as espécies de eucalipto estudadas, os valores médios de ligação interna estão acima do valor mínimo de referência exigido pela norma americana CS 236-66. Os resultados também foram satisfatórios com relação aos painéis produzidos com Pinus elliottii, Mimosa scabrella e Eucaliptus dunnii, espécies mencionadas na literatura;

4) Para painéis produzidos com $8 \%$ de resina, o Eucalyptus maculata apresentou maior valor médio de ligação interna;

5) Para $12 \%$ de resina, o maior valor foi obtido com Eucalyptus tereticornis;

6) O módulo de elasticidade dos painéis de Eucalyptus maculata e Eucalyptus grandis foi superior ao dos painéis produzidos com Eucalyptus tereticornis;

7) Não foi constatada diferença estatisticamente significativa entre os painéis produzidos com 8 e $12 \%$ de resina;

8) Para o módulo de ruptura, os painéis de Eucalyptus maculata, Eucalyptus grandis e a mistura das três espécies estudadas apresentaram valores médios superiores em relação aos painéis de Eucalyptus tereticornis;

9) Os painéis produzidos com $12 \%$ de resina apresentaram MOR superior aos painéis com $8 \%$;

10) Em relação às propriedades mecânicas e em comparação com os resultados de pesquisas anteriores tem-se que, entre as espécies estudadas, o Eucalyptus maculata e o Eucalyptus grandis foram as que apresentaram melhor comportamento para produção de painéis de madeira aglomerada; e

11) A espécie Eucalyptus tereticornis poderá ser utilizada em mistura com as demais espécies estudadas.

\section{REFERÊNCIAS}

1 AMERICAN SOCIETY FOR TESTING AND MATERIALS. Standard methods of evaluating properties of wood-base fiber and particle materials. In: Annual Book of ASTM Standards, ASTM D-1037 - 78B. Philadelphia, 1992.

2 BRITO, E.O. Estimativa da produção de resíduos na indústria brasileira de serraria e laminação de madeira. Revista da Madeira, Caxias do Sul, v.26, p.34-39, 1996.

3 COMMERCIAL STANDARD. Mat formed wood particleboard. CS 236-66. 1968.

4 EL-OSTA, M.L.M.; E.L-MORSHEDY, M.M.; MEGAHED, M.M. Properties of particleboard made from Casuarina and Willow mixtures. Proceedings of $\mathbf{2 8}^{\text {th }}$ internacional particleboard /composite materials symposium, Pullmann, 1994, p.281288. 
IWAKIRI, S. et. al. Resíduos de serrarias...

5 IMAMURA, Y. Estimation of the fungal resistance of wood composites of structural use - Recent research on wood and wood-based materials. Current Japanese Material Research. Elsevier Applied Science, London, v.11, p.75-84, 1993.

6 IWAKIRI, S.; KLOCK, U.; ROCHA, M.P.; SEVERO, E.T.D.; RINCOSKI, C.R.; BORTOLETTO JUNIOR, G.; PIO, N.S. Mistura de espécies na produção de chapas de partículas estruturais "waferboard". Revista do Setor de Ciências Agrárias, Curitiba, v.14, n.1-2, p.107-114, 1995.

7 IWAKIRI, S.; LATORRACA, J.V.F.; SILVA, D.A.; GABARDO, J.L.; KLITZKE, R.J.; FOFANO JUNIOR, A.; FABROWSKI, F.; INTERAMENSE, M.T. Produção de chapas de partículas de madeira aglomerada de Pinus elliottii (Engelm) e Eucalyptus dunnii (Maid). Revista do Setor de Ciências Agrárias, Curitiba, v.15, n.1, p.33-41, 1996.

8 KAWAI, S.; SASSAKI, H. Low density particleboard. Recent research on wood and wood-based materials. Current Japanese materials research. Elsevier Applied Science, London, v.11, p.33-41, 1993.

9 KELLY, M.W. Critical literature review of relationships between processing parameters and physical properties of particleboard. U.S. Despartment of Agriculture. Wisconsin: FPL, 1977. 66p.

10 MALONEY, T.M. Modern particleboard \& dry process fiberboard manufacturing. San Francisco: Miller Freeman Inc. 1993. 681p.

11 MOSLEMI, A.A. Particleboard. Illinois: Southern Illinois University Press, 1974. 244p.

12 TSOUMIS, G. Science and technology of wood. Structure, properties, utilization. New York: Chapman \& Hall, 1991. 494p. 